Efigênia Aparecida Maciel de Freitas'

Ismênia Diniz Mendes"

Luiz Carlos Marques de Oliveira"II

Universidade Presidente Antônio Carlos. Araguari, MG, Brasil

" Curso de Graduação em Medicina. Faculdade de Medicina (Famed). Universidade Federal de Uberlândia (UFU). Uberlândia, MG, Brasil

III Departamento de Clínica Médica. Famed. UFU. Uberlândia, MG, Brasil

Correspondência | Correspondence: Luiz Carlos Marques de Oliveira

R. Gonçalves Dias, 455 - Bairro Tabajaras

38400-288 Uberlândia, MG, Brasil

E-mail: oliveiralcm@ufu.br

\section{Ingestão alcoólica em vítimas de causas externas atendidas em um hospital geral universitário}

\section{Alcohol consumption among victims of external causes in a university general hospital}

\section{RESUMO}

OBJETIVO: Estimar a freqüência de ingestão alcoólica em vítimas de causas externas atendidas em hospital.

MÉTODOS: Estudo realizado com vítimas atendidas em um hospital geral universitário em Uberlândia (MG), de fevereiro a agosto de 2004. A alcoolemia foi determinada em 85 pacientes no pronto-socorro e entrevistaram-se outros 301 internados nas enfermarias sobre possível ingestão alcoólica previamente ao trauma; em ambos os grupos foi aplicado o questionário Cut-down, Annoyed by criticism, Guilty and Eye-opener (CAGE). Para as comparações das freqüências foi utilizado o teste exato de Fisher.

RESULTADOS: A alcoolemia foi positiva em 31,8\% dos pacientes testados, os quais mais freqüentemente necessitaram de internação (70,4\% versus $37,9 \% ; p<0,05)$. Proporcionalmente, alcoolemia positiva foi mais freqüente $(\mathrm{p}<0,05)$ entre as vítimas de agressão física $(57,1 \%)$ do que as de queda $(18,2 \%)$ ou de acidente de trânsito (29,3\%). Nas enfermarias, $29,9 \%$ dos pacientes referiram ingestão alcoólica, proporcionalmente mais freqüente $(p<0,01)$ entre as vítimas de agressão física $(67,4 \%)$ do que entre as de acidente de trânsito (27,8\%) ou queda (19,3\%). Entre aqueles que ingeriram álcool, abordados no pronto-socorro e nas enfermarias, observou-se, respectivamente: que a maioria era homens (85,2\% e $80,4 \%)$, a ocorrência de trauma foi maior $(\mathrm{p}<0,05)$ nos finais de semana (63\% e $57,8 \%$ ) e no período noturno (59,3\% e $57,8 \%$ ), e o questionário CAGE foi positivo em $81,5 \%$ e $82,2 \%$.

CONCLUSÕES: Cerca de um terço dos pacientes ingeriu bebidas alcoólicas previamente ao trauma e, entre eles, a maioria era homens. Proporcionalmente, a ingestão prévia de bebidas alcoólicas foi mais freqüente entre os pacientes vítimas de violência. Os resultados da aplicação do CAGE mostra que a maioria dos pacientes vítimas de causas externas após ingestão etílica não era alcoolista ocasional, e sim provável usuário crônico ou dependente de álcool.

DESCRITORES: Consumo de Bebidas Alcoólicas. Intoxicação Alcoólica. Agressão. Acidentes de Trânsito. Acidentes por Quedas. Causas Externas. 


\begin{abstract}
OBJECTIVE: To estimate frequency of alcohol consumption among victims of external causes cared for in a hospital.

METHODS: Study performed with victims cared for in a university general hospital in the city of Uberlândia (Southeastern Brazil), between February and August 2004. Blood alcohol content was determined from 85 patients in the emergency room. Other 301 patients, hospitalized in the outpatient clinics, were interviewed about possible alcohol consumption prior to trauma. The Cut-down, Annoyed by criticism, Guilty and Eye-opener (CAGE) questionnaire was applied in both groups. Fisher's exact test was employed to compare frequencies.
\end{abstract}

RESULTS: Blood alcohol content was positive among 31.8\% of patients tested, who most frequently required hospitalization $(70.4 \%$ versus $37.9 \%$; $\mathrm{p}<0.05)$. Proportionately, positive blood alcohol content was more frequent $(\mathrm{p}<0.05)$ among victims of physical aggression $(57.1 \%)$ than fall victims (18.2\%) or those of traffic accidents (29.3\%). In outpatient clinics, $29.9 \%$ of patients mentioned alcohol consumption, proportionately more frequent $(p<0.01)$ among victims of physical aggression $(67.4 \%)$ than traffic accident victims (27.8\%) or fall victims (19.3\%). Among those who had drunk alcohol and were approached in the emergency room or in the outpatient clinics, the following was observed: the majority was male (85.2\% and $80.4 \%)$, the occurrence of trauma was higher $(\mathrm{p}<0.05)$ on weekends $(63 \%$ and $57.8 \%)$ and at night (59.3\% and 57.8\%), and the CAGE questionnaire was positive among $81.5 \%$ and $82.2 \%$ of them.

CONCLUSIONS: About a third of patients had drunk alcoholic beverages prior to trauma and the majority of them were male. Proportionately, previous alcohol consumption was more frequent among patients who were victims of violence. CAGE results show that most patients, victims of external causes after alcohol consumption, were not occasional drinkers, but probably chronic users or alcoholics.

DESCRIPTORS: Alcohol Drinking. Alcoholic Intoxication. Aggression. Accidents, Traffic. Accidental Falls. External Causes.

\section{INTRODUÇÃO}

O uso abusivo de bebidas alcoólicas constitui importante problema de saúde pública em muitos países. No Brasil, em dois levantamentos nacionais, estimou-se que $9 \%{ }^{\mathrm{a}}$ a $12,3 \%^{3}$ da população sejam dependentes do álcool; $74,6 \%$ já fizeram uso na vida (80,4\% na região Sudeste), ${ }^{3}$ $29 \%$ são bebedores pouco freqüentes e não fazem uso pesado e $24 \%$ bebem freqüentemente e pesado. ${ }^{\text {a }}$ Vários são os fatores que podem levar ao aumento do consumo de bebidas alcoólicas na população, entre eles, a fácil disponibilidade, o baixo preço e a publicidade. ${ }^{2}$ Além disso, a ingestão alcoólica é aceita socialmente, algumas vezes mesmo em quantidades consideradas abusivas, sendo utilizada como um facilitador de atividades interpessoais, em estabelecer vínculos sociais, e pode fazer parte do código de polidez em vários contextos. ${ }^{17}$
O consumo abusivo e/ou inadequado desta substância pode trazer conseqüências graves, tanto em nível orgânico, como psicológico e social. Entre estas conseqüências estão as ocorrências de lesões por causas externas, sobretudo acidentes ou violência, conceituadas pela "Política Nacional de Redução da Morbi-mortalidade por Acidentes e Violência” como o conjunto das ocorrências que matam ou geram agravos à saúde e que demandam atendimento nos serviços de saúde. Acidente é entendido como o "evento não intencional e evitável, causador de lesões físicas e/ou emocionais”, e violência o "evento representado por ações realizadas por indivíduos, grupos, nações, classes, que ocasionam danos físicos, emocionais, morais e/ou espirituais a si próprio ou a outros". ${ }^{\mathrm{b}}$

a Laranjeira R, Pinsky I, Zaleski M, Caetano R. I levantamento nacional sobre os padrões de consumo de álcool na população brasileira [internet] Brasília: Secretaria Nacional Antidrogas; 2007 [citado 2008 ago 3]. Disponível em: http://www.senad.gov.br/relatorio_padroes_ consumo_alcool.pdf

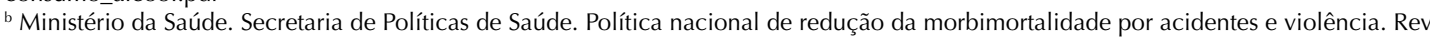
Saude Publica. 2000;34(4):427-30. 
Estudo multicêntrico com vítimas de causas externas não-fatais, envolvendo 10 países, mostrou que 18,1\% tinham ingerido bebidas alcoólicas durante as seis horas que precederam o evento, e esta freqüência foi mais baixa no Canadá (6\%) e mais alta na Nova Zelândia (38,5\%). ${ }^{1} \mathrm{Na}$ Europa, acredita-se que o álcool esteja implicado em 40\%-60\% dos casos de lesões não intencionais e violência. ${ }^{22}$ Alguns estudos têm sido desenvolvidos no Brasil para avaliar a relação entre o abuso de bebidas alcoólicas e a ocorrências de causas externas, e geralmente são realizados nas capitais de estados ou em suas regiões metropolitanas. Em São Paulo (SP), alcoolemias positivas foram observadas em $11 \%$ dos pacientes vítimas de traumas não-fatais atendidos em um pronto-socorro, ${ }^{21}$ em $28,9 \%$ dos pacientes atendidos em um centro de atenção ao trauma, vítimas de acidentes de transporte, de quedas, agressões e outros, ${ }^{11} \mathrm{e}$ em aproximadamente metade das vítimas fatais de causas externas durante o ano de $1994 .{ }^{4}$

O abuso de bebidas alcoólicas tem importante impacto nos índices de violência. Na cidade de São Paulo, 42,5\% das vítimas de homicídios submetidas a exame toxicológico tinham feito uso de álcool, ${ }^{10}$ e em Curitiba (PR), as vítimas de agressões interpessoais e por arma de fogo e arma branca, atendidas em um pronto-socorro, estavam alcoolizadas em 50,2\% dos casos. ${ }^{12}$

O objetivo do presente estudo foi estimar a freqüência de ingestão prévia de bebidas alcoólicas entre as vítimas de lesões por causas externas atendidas em hospital.

\section{MÉTODOS}

Estudo realizado em um hospital geral universitário, de referência regional e conveniado ao Sistema Único de Saúde (SUS), em Uberlândia, município do interior do Estado de Minas Gerais, no período de fevereiro a agosto de 2004. A população estimada pelo Instituto Brasileiro de Geografia e Estatística para o ano de 2006 era de 600.368 habitantes, com expressiva expansão urbana. Segundo dados do Ministério da Saúde, foram gastos R\$ 4.605.089,59 em 3.494 internações em decorrência de causas externas no ano de 2005.

Foram incluídos pacientes vítimas de lesões por causas externas, com idade mínima de 18 anos, atendidos no pronto-socorro ou internados nas enfermarias de cirurgia ou traumatologia.

No pronto-socorro, foram avaliados 85 pacientes à sua admissão no setor de emergência. Eles foram entrevistados com base em um questionário que continha perguntas referentes às suas características e condições socioeconômicas, tais como, sexo, idade, escolaridade, estado civil, renda familiar e informações relativas ao evento que motivou a procura pelo hospital. Também foi aplicado a cada um dos pacientes o questionário Cut-down, Annoyed by criticism, Guilty e Eye-opener
(CAGE) para diagnóstico de dependência ou abuso crônico do álcool, sendo considerado CAGE positivo quando havia duas ou mais respostas afirmativas às suas quatro perguntas. ${ }^{9}$ Em seguida, coletaram-se amostras de sangue de cada um dos pacientes que concordaram em participar do estudo para a determinação da alcoolemia. Avaliou-se também a evolução de cada caso: alta, internação ou óbito.

As entrevistas foram realizadas pelos autores em plantões diurnos de seis horas, das 6:30 às 12:30 ou de 12:30 às 18:30, e plantões noturnos de 12 horas, ou seja, entre 18:30 e 6:30. Os plantões diurnos e noturnos foram realizados em dias alternados e, após sorteio, o primeiro plantão ocorreu em uma terça-feira no período matutino. O final de semana foi o período compreendido entre as 18:00 de sexta feira e 6:00 de segunda feira.

As amostras de sangue foram coletadas em tubos de vidro com gel separador e, após a centrifugação, o soro foi armazenado a $-20^{\circ} \mathrm{C}$. Para a determinação das alcoolemias, utilizou-se o kit TDx/TDxFLx-Etanol (Abbott, Abbott Park, IL, USA); foram consideradas positivas as amostras que contivessem alcoolemia em concentração igual ou superior a $0,1 \mathrm{~g} / \mathrm{l}$ de sangue, de acordo com as orientações do fabricante do kit utilizado. Para preservar o sigilo da identidade dos pacientes, as amostras, assim como as entrevistas, foram identificadas apenas com numeração cardinal.

Nas enfermarias de cirurgia geral ou de traumatologia foram avaliados, consecutivamente, 301 pacientes hospitalizados em decorrência de lesões por causas externas; foram excluídos aqueles que já haviam sido entrevistados no pronto-socorro e nenhum dos pacientes abordados se recusou a participar do estudo. Os pacientes foram indagados: sobre as suas condições socioeconômicas; sobre o trauma que motivou a sua internação; e se havia história de ingestão de bebidas alcoólicas previamente ao evento. Também foi aplicado o questionário CAGE a todos os pacientes estudados. Considerou-se história positiva de ingestão de bebidas alcoólicas quando havia relato de consumo de pelo menos uma garrafa de cerveja $(660 \mathrm{ml})$ ou duas doses (100ml) de destilados (uísque, vodca ou cachaça) equivalentes a $25 \mathrm{~g}$ e $32 \mathrm{~g}$ de etanol, respectivamente, ${ }^{18}$ até seis horas antes do trauma. A ingestão de aproximadamente 44 g produz concentrações de $0,4 \mathrm{~g} / \mathrm{l}$ a $0,9 \mathrm{~g} / \mathrm{l}$ de sangue, dependendo do tipo de bebida e da ingestão prévia ou não de alimentos. ${ }^{19}$

Não foram incluídos pacientes com menos de 18 anos de idade. Foram excluídos também dois pacientes do pronto-socorro que não podiam se comunicar por estarem com entubação oro-traqueal e desacompanhados, e outros três que não concordaram em participar. O diagnóstico do tipo de lesão por causa externa foi sempre realizado pelo médico responsável pelo paciente. 
Para as comparações das freqüências analisadas foram utilizados os testes do qui-quadrado ou o exato de Fisher, e considerou-se como significante $\mathrm{p}<0,05$.

O projeto deste estudo foi aprovado pelo comitê de ética em pesquisa institucional e de cada paciente ou de seu familiar foi obtido o consentimento informado para sua participação.

\section{RESULTADOS}

Na Tabela 1 observam-se os dados dos 85 pacientes avaliados no pronto-socorro: 67 (78,8\%) eram do sexo masculino e 18 (21,2\%) do sexo feminino, com média de idade e desvio-padrão (DP) de 34,8 $\pm 13,1$ anos; a faixa etária predominante foi a de 18 a 25 anos (30,6\%). Na sua maioria, os pacientes tinham no máximo o ensino fundamental $(62,4 \%)$, não eram casados $(62,3 \%)$ e tinham renda mensal de até três salários mínimos (77,6\%). O tipo de causa externa mais freqüente foi acidente de trânsito (68,2\%), seguido de agressão física $(16,5 \%)$, quedas $(12,9 \%)$ e outros $(2,4 \%)$.

A alcoolemia foi positiva em 27 (31,8\%) pacientes, em 24 (28,2\%) foi superior a 0,6g/l; a faixa etária predominante foi de 31 a 40 anos (37\%), a maioria era do sexo masculino (85,2\%), escolaridade máxima até o ensino fundamental (81,5\%), era não casada (63\%) e tinha renda mensal de até três salários mínimos (96,3\%). A freqüência de pacientes com escolaridade de ensino médio ou superior, ou com renda familiar superior a três salários mínimos foi maior $(\mathrm{p}<0,05)$ entre aqueles com alcoolemia negativa (31,8\% e 21,2\%, respectivamente) do que entre os pacientes com alcoolemia positiva (5,9\% e 1,2\%, respectivamente). A freqüência de alcoolemia positiva, proporcionalmente, foi semelhante entre os sexos masculino $(34,3 \%)$ e feminino $(22,2 \%)$ e entre os casados (32,1\%) e não-casados (31,2\%).

Proporcionalmente, a alcoolemia positiva foi mais freqüente $(\mathrm{p}<0,05)$ entre as vítimas de agressão física $(57,1 \%)$ do que as de queda $(18,2 \%)$, e tendeu $(p>0,05)$ a ser também mais freqüente do que as de acidente de trânsito (29,3\%) (Tabela 3).

Entre os pacientes com alcoolemia positiva, os tipos de lesões mais freqüentes foram as corto-contusas (30,2\%) e as fraturas (25,6\%), o trauma na cabeça foi observado em 34,5\% e foi observado entre eles o único caso de amputação (membro inferior esquerdo). Ainda entre

Tabela 1. Características dos pacientes atendidos no pronto-socorro segundo positividade de alcoolemia e aos resultados do questionário CAGE. Uberlândia, MG, 2004. (n=85)

\begin{tabular}{|c|c|c|c|c|c|c|c|c|c|c|}
\hline \multirow{3}{*}{ Variável } & \multicolumn{4}{|c|}{ Alcoolemia } & \multicolumn{4}{|c|}{ CAGE } & & \\
\hline & \multicolumn{2}{|c|}{ Positiva } & \multicolumn{2}{|c|}{ Negativa } & \multicolumn{2}{|c|}{ Positivo } & \multicolumn{2}{|c|}{ Negativo } & \multicolumn{2}{|c|}{ Total } \\
\hline & $\mathrm{n}$ & $\%$ & $\mathrm{n}$ & $\%$ & $\mathrm{n}$ & $\%$ & $\mathrm{n}$ & $\%$ & $\mathrm{n}$ & $\%$ \\
\hline \multicolumn{11}{|l|}{ Sexo } \\
\hline Masculino & 23 & 27,0 & 44 & 51,8 & 32 & 37,6 & 35 & 41,2 & 67 & 78,8 \\
\hline Feminino & 4 & 4,7 & 14 & 16,4 & 4 & 4,7 & 14 & 16,5 & 18 & 21,2 \\
\hline Total & 27 & 31,8 & 58 & 68,2 & 36 & 42,4 & 49 & 57,6 & 85 & 100 \\
\hline \multicolumn{11}{|l|}{ Faixa etária (anos) } \\
\hline $18 I-25$ & 4 & 4,7 & 22 & 25,9 & 7 & 8,2 & 19 & 22,4 & 26 & 30,6 \\
\hline $25 I-30$ & 3 & 3,5 & 12 & 14,1 & 5 & 5,9 & 10 & 11,8 & 15 & 17,6 \\
\hline $30 \mid-40$ & 10 & 11,7 & 6 & 7,1 & 13 & 15,3 & 3 & 3,5 & 16 & 18,8 \\
\hline $40 \mathrm{I}-50$ & 5 & 5,9 & 10 & 11,7 & 7 & 8,2 & 8 & 9,4 & 15 & 17,6 \\
\hline$\geq 50$ & 5 & 5,9 & 8 & 9,4 & 4 & 4,7 & 9 & 10,6 & 13 & 15,3 \\
\hline \multicolumn{11}{|l|}{ Escolaridade } \\
\hline$\leq$ Fundamental & 22 & 25,9 & 31 & 36,5 & 29 & 34,1 & 24 & 28,2 & 53 & 62,4 \\
\hline$\geq$ Médio & 5 & 5,9 & 27 & 31,8 & 7 & 8,2 & 25 & 29,4 & 32 & 37,6 \\
\hline \multicolumn{11}{|l|}{ Estado civil } \\
\hline Casado & 10 & 11,7 & 22 & 25,9 & 12 & 14,1 & 20 & 23,5 & 32 & 37,6 \\
\hline Não-casado* & 17 & 20,0 & 36 & 42,3 & 24 & 28,2 & 29 & 34,1 & 53 & 62,3 \\
\hline \multicolumn{11}{|l|}{ Renda** } \\
\hline$\leq 3$ & 26 & 30,6 & 40 & 47,0 & 35 & 41,2 & 31 & 36,5 & 66 & 77,6 \\
\hline$>3$ & 1 & 1,2 & 18 & 21,2 & 1 & 1,2 & 18 & 21,2 & 19 & 22,4 \\
\hline
\end{tabular}

CAGE: Cut-down, Annoyed by criticism, Guilty and Eye-opener

* solteiros $(n=43)$, separados $(n=6)$, desquitados/divorciados $(n=3)$, viúvo $(n=1)$;

** renda familiar em salários mínimos. 
os pacientes com alcoolemia positiva, a freqüência de acidentes nos finais de semana $(63 \%$; $\mathrm{p}<0,01)$ e no período noturno $(59,3 \%$; $\mathrm{p}<0,05)$ foi maior do que entre os pacientes com alcoolemia negativa, em que a maior freqüência de acidentes ocorreu no período diurno $(62,1 \%)$ e nos dias úteis da semana $(70,7 \%)$.

Dos 85 pacientes do pronto-socorro, 36 (42,4\%) apresentaram CAGE positivo, mais freqüente $(\mathrm{p}<0,05)$ no sexo masculino $(88,9 \%)$ do que no feminino $(11,1 \%)$. As freqüências de escolaridade com níveis de ensino médio ou superior e de renda familiar acima de três salários mínimos foram maiores $(\mathrm{p}<0,01)$ no subgrupo de pacientes com CAGE negativo $(29,4 \%)$ do que naquele com CAGE positivo $(8,2 \%)$. Entre os pacientes com CAGE positivo o tipo de causa externa proporcionalmente mais freqüente $(\mathrm{p}<0,05)$ foi a agressão física (64,3\%) em relação ao acidente de trânsito (34,5\%); não se observaram diferenças significantes entre as freqüências de queda (63,6\%) e agressão ou entre queda e acidente de trânsito (Tabela 3). Dos 36 pacientes com CAGE positivo, $22(61,1 \%)$ tinham alcoolemia positiva e 14 (38,9\%) alcoolemia negativa; entre os 49 pacientes com CAGE negativo, cinco (10,2\%) tinham alcoolemia positiva e $44(89,8 \%)$ negativa $(\mathrm{p}<0,01)$. A freqüência de questionário CAGE positivo foi maior $(\mathrm{p}<0,01)$ entre os pacientes com alcoolemia positiva $(81,5 \%)$ do que entre aqueles com alcoolemia negativa $(24,1 \%)$. As quatro pacientes do sexo feminino com alcoolemia positiva tinham CAGE positivo.

Quanto à evolução após atendimento na sala de emergência, a freqüência de necessidade de internação foi maior $(\mathrm{p}<0,05)$ entre os pacientes com alcoolemia positiva $(19 / 27 ; 70,4 \%)$ do que entre aqueles com alcoolemia negativa (22/58; 37,9\%), e nenhum dos pacientes avaliados foi a óbito.

Dos 301 pacientes hospitalizados nas enfermarias em decorrência de lesões por causas externas, a maioria era do sexo masculino $(80,4 \%)$, com escolaridade até o ensino fundamental $(72,8 \%)$, com renda familiar de até três salários mínimos (62,8\%), sem predomínio de nenhuma das faixas etárias avaliadas (Tabela 2). Entre eles, 90 (29,9\%) relataram ingestão de bebidas alcoólicas antes do trauma, e a quantidade mínima ingerida foi de duas garrafas de $660 \mathrm{ml}$ de cerveja e duas doses de destilados. Entre os pacientes que

Tabela 2. Características dos pacientes hospitalizados nas enfermarias de cirurgia e traumatologia segundo história de ingestão de bebida alcoólica e resultados do questionário CAGE. Uberlândia, MG, 2004. (n=301)

\begin{tabular}{|c|c|c|c|c|c|c|c|c|c|c|}
\hline \multirow{3}{*}{ Variável } & \multicolumn{6}{|c|}{ História de ingestão de bebida alcoólica } & \multicolumn{4}{|c|}{ CAGE } \\
\hline & \multicolumn{2}{|c|}{ Positiva } & \multicolumn{2}{|c|}{ Negativa } & \multicolumn{2}{|c|}{ Positivo } & \multicolumn{2}{|c|}{ Negativo } & \multicolumn{2}{|c|}{ Total } \\
\hline & $\mathrm{n}$ & $\%$ & $\mathrm{n}$ & $\%$ & $\mathrm{n}$ & $\%$ & $\mathrm{n}$ & $\%$ & $\mathrm{n}$ & $\%$ \\
\hline \multicolumn{11}{|l|}{ Sexo } \\
\hline Masculino & 84 & 27,9 & 158 & 52,5 & 110 & 36,5 & 132 & 43,9 & 242 & 80,4 \\
\hline Feminino & 6 & 2,0 & 53 & 17,6 & 10 & 3,3 & 49 & 16,3 & 59 & 19,6 \\
\hline Total & 90 & 29,9 & 211 & 70,1 & 120 & 39,9 & 181 & 60,1 & 301 & 100 \\
\hline \multicolumn{11}{|l|}{ Faixa etária (anos) } \\
\hline $18 \mathrm{I}-25$ & 20 & 6,6 & 40 & 13,3 & 28 & 9,3 & 32 & 10,6 & 60 & 19,9 \\
\hline $25 \mathrm{I}-30$ & 20 & 6,6 & 30 & 10,0 & 20 & 6,6 & 30 & 10,0 & 50 & 16,6 \\
\hline $30 I-40$ & 24 & 8,0 & 35 & 11,6 & 30 & 10,0 & 29 & 9,6 & 59 & 19,6 \\
\hline $40 I-50$ & 16 & 5,3 & 37 & 12,3 & 20 & 6,6 & 33 & 11,0 & 53 & 17,6 \\
\hline$\geq 50$ & 10 & 3,3 & 69 & 22,9 & 22 & 7,3 & 57 & 18,9 & 79 & 26,2 \\
\hline \multicolumn{11}{|l|}{ Escolaridade } \\
\hline$\leq$ Fundamental & 73 & 24,3 & 146 & 48,5 & 92 & 30,6 & 127 & 42,2 & 219 & 72,8 \\
\hline$\geq$ Médio & 17 & 5,6 & 65 & 21,6 & 28 & 9,3 & 54 & 17,9 & 82 & 27,2 \\
\hline \multicolumn{11}{|l|}{ Estado civil } \\
\hline Casado & 41 & 13,6 & 91 & 30,2 & 45 & 14,9 & 87 & 28,9 & 132 & 43,8 \\
\hline Não-casado* & 49 & 16,3 & 120 & 39,9 & 72 & 23,9 & 97 & 32,2 & 169 & 56,2 \\
\hline \multicolumn{11}{|l|}{ Renda** } \\
\hline$\leq 3$ & 57 & 18,9 & 132 & 43,8 & 84 & 27,9 & 105 & 34,9 & 189 & 62,8 \\
\hline$>3$ & 33 & 11,0 & 79 & 26,2 & 26 & 8,6 & 86 & 28,6 & 112 & 37,2 \\
\hline
\end{tabular}

CAGE: Cut-down, Annoyed by criticism, Guilty and Eye-opener

* solteiro $(n=33)$, separado $(n=9)$, desquitado/divorciado $(n=5)$, viúvo $(n=2)$;

** renda familiar em salário mínimo vigente. 
relataram ingestão alcoólica, a maioria $(93,3 \%)$ era do sexo masculino $(\mathrm{p}<0,01)$, com escolaridade até o ensino fundamental $(81,1 \%)$, com renda familiar de até três salários mínimos (63,3\%) e de não-casados (55,4\%). A freqüência de escolaridade de níveis médio ou superior foi maior $(p<0,05)$ entre os pacientes que relataram não ter ingerido álcool $(30,8 \%)$ do que os que relataram positivamente (18,9\%). Proporcionalmente, a ingestão alcoólica foi mais freqüente $(\mathrm{p}<0,01)$ entre os pacientes vítimas de agressão física $(67,4 \%)$ do que entre aqueles vítimas de acidentes de trânsito $(27,8 \%)$ ou queda (19,3\%). (Tabela 3 )

Os traumatismos raquimedulares ou cranianos foram mais freqüentes $(\mathrm{p}<0,05)$ entre os pacientes que relataram ingestão alcoólica (66,6\% e 37,6\%, respectivamente) do que entre aqueles com história negativa (33,3\% e $10,9 \%$, respectivamente).

O questionário CAGE foi positivo em 120 (39,9\%) pacientes (Tabela 2). CAGE positivo foi mais freqüente $(p<0,01)$ entre as vítimas de agressão física $(74,4 \%)$ em relação às de acidentes de trânsito (40,7\%) (Tabela 3), no sexo masculino $(91,7 \%)$ e entre aqueles com renda familiar menor do que três salários mínimos (Tabela 2). Destes pacientes com CAGE positivo, 74 (61,7\%) relataram ingestão alcoólica prévia ao acidente e 46 $(38,3 \%)$ relataram não ter ingerido álcool $(\mathrm{p}<0,01)$. A freqüência de questionário CAGE positivo foi maior $(\mathrm{p}<0,01)$ entre os pacientes que relataram ingestão alcoólica (82,2\%) do que entre aqueles que relataram não ter ingerido álcool (21,8\%).

Entre os pacientes que relataram ingestão alcoólica prévia, a freqüência de acidentes nos finais de semana $(57,8 \% ; \mathrm{p}<0,01)$ e no período noturno $(57,8 \% ; \mathrm{p}<0,05)$ foi maior do que entre aqueles que relataram não ter ingerido álcool, em que a maior ocorrência de acidentes foi no período diurno $(69,2 \%)$ e nos dias úteis $(64 \%)$.

\section{DISCUSSÃO}

Apesar dos diferentes métodos de avaliação empregados para o diagnóstico de ingestão alcoólica em vítimas de causas externas, no pronto-socorro e nas enfermarias, os resultados obtidos foram semelhantes. A freqüência de alcoolemia positiva observada no pronto-socorro foi semelhante àquela encontrada em vítimas de causas externas admitidas em um centro urbano de atenção ao trauma no município de São Paulo $(28,9 \%) .{ }^{11}$ Entretanto, freqüência maior foi observada entre as vítimas fatais de causas externas, também na área metropolitana de São Paulo (48,3\%). ${ }^{4}$ As freqüências de alcoolemias acima de $0,6 \mathrm{~g} / \mathrm{l}$ encontradas no presente estudo foram semelhantes àquelas observadas em um hospital de pronto-socorro em Porto Alegre (RS), onde alcoolemias acima de $0,8 \mathrm{~g} / \mathrm{l}$ foram observadas em 24,5\% das vítimas de acidentes de trânsito. ${ }^{5}$ Freqüência menor de diagnóstico de ingestão alcoólica (18,9\%) foi verificada entre as vítimas de causas externas em um pronto-socorro de Curitiba, quando se utilizou para este diagnóstico evidências clínicas de embriaguez e relato de ingestão alcoólica pelo paciente. ${ }^{12}$

No presente estudo, verificou-se entre os pacientes com alcoolemia positiva uma freqüência elevada de níveis séricos acima de 0,6g/l; alcoolemias a partir de 0,5g/l podem provocar incoordenação motora, alteração do humor, da personalidade e do comportamento, podendo comprometer o desempenho de atividades rotineiras. Essas manifestações podem se intensificar conforme o aumento da concentração sérica de etanol, ${ }^{16,19}$ predispondo o indivíduo à ocorrência de acidentes ou de violência.

Houve maior prevalência de alcoolemia positiva, assim como de história de ingestão alcoólica prévia ao trauma, entre os indivíduos do sexo masculino, não

Tabela 3. Tipo de ocorrência de causa externa entre os pacientes atendidos no pronto-socorro e nas enfermarias de cirurgia e traumatologia, segundo positividade à alcoolemia, ingestão alcoólica e os resultados do questionário CAGE. Uberlândia, MG, 2004. $(\mathrm{N}=386)$

\begin{tabular}{|c|c|c|c|c|c|c|c|c|c|c|}
\hline \multirow{3}{*}{ Tipo de causa externa } & \multicolumn{4}{|c|}{ Alcoolemia/História prévia de ingestão alcoólica } & \multicolumn{4}{|c|}{ CAGE } & \multirow{2}{*}{\multicolumn{2}{|c|}{ Total }} \\
\hline & \multicolumn{2}{|c|}{ Positiva } & \multicolumn{2}{|c|}{ Negativa } & \multicolumn{2}{|c|}{ Positivo } & \multicolumn{2}{|c|}{ Negativo } & & \\
\hline & $\mathrm{n}$ & $\%$ & $\mathrm{n}$ & $\%$ & $\mathrm{n}$ & $\%$ & $\mathrm{n}$ & $\%$ & $\mathrm{n}$ & $\%$ \\
\hline \multicolumn{11}{|l|}{ Pronto-socorro $(n=85)$} \\
\hline Trânsito & 17 & 29,3 & 41 & 70,7 & 20 & 34,5 & 38 & 65,5 & 58 & 100 \\
\hline Agressões & 8 & 57,1 & 6 & 42,9 & 9 & 64,3 & 5 & 35,7 & 14 & 100 \\
\hline Quedas & 2 & 18,2 & 9 & 81,8 & 7 & 63,6 & 4 & 36,4 & 11 & 100 \\
\hline Outros & 0 & 0 & 2 & 100 & 0 & 0 & 2 & 100 & 2 & 100 \\
\hline \multicolumn{11}{|l|}{ Enfermarias $(n=301)$} \\
\hline Trânsito & 45 & 27,8 & 117 & 72,2 & 66 & 40,7 & 96 & 59,3 & 162 & 100 \\
\hline Agressões & 29 & 67,4 & 14 & 32,6 & 32 & 74,4 & 11 & 25,6 & 43 & 100 \\
\hline Quedas & 16 & 19,3 & 67 & 80,7 & 19 & 22,9 & 64 & 77,1 & 83 & 100 \\
\hline Outros & 0 & 0 & 13 & 100 & 3 & 23,1 & 10 & 76,9 & 13 & 100 \\
\hline
\end{tabular}

CAGE: Cut-down, Annoyed by criticism, Guilty and Eye-opener 
casados e com baixos níveis de escolaridade e de renda familiar. Este mesmo perfil de pacientes foi verificado em estudos realizados em outras cidades brasileiras. Nesses estudos, observou-se que as pessoas que se envolvem em acidentes, inclusive em decorrência de consumo de álcool, são freqüentemente homens, ${ }^{10,11,13,15}$ da faixa etária economicamente ativa e com baixa escolaridade. ${ }^{11}$ Em um levantamento nacional observou-se que os homens se expõem a riscos físicos sob efeito do álcool três vezes mais do que as mulheres; ${ }^{3}$ em outro estudo, a freqüência de beber e dirigir foi 7,4 vezes maior entre os homens do que entre as mulheres. ${ }^{a}$ Isso pode ser justificado pelo fato de que os homens adotam comportamento e atividades de risco mais freqüentemente do que as mulheres, ${ }^{5,13}$ e ainda, que no Brasil, por razões socioculturais, os homens abusam da ingestão alcoólica com mais freqüência do que as mulheres. ${ }^{\mathrm{a}, 3}$ A predominância de indivíduos com baixos níveis de escolaridade e de renda familiar, observada no presente estudo, também pode se relacionar ao fato de que o hospital onde este estudo foi realizado é um serviço de referência para a rede pública de saúde do município e região, atendendo a parcela da população que não dispõe de outro tipo de assistência à saúde.

A observação de maior freqüência, proporcionalmente, de alcoolemia positiva ou de história de ingestão etílica prévia ao trauma, ou ainda, de CAGE positivo entre os pacientes vítimas de agressão, mostra que indivíduos alcoolizados e/ou etilistas crônicos estão mais predispostos à violência. Esses resultados são semelhantes àqueles observados em um estudo realizado em São Paulo, no qual se verificou maior freqüência de alcoolemia positiva entre as vítimas de agressão (46,2\%) do que entre as de acidente de transporte $(24,2 \%)$ ou de queda (20,2\%). ${ }^{11}$ Em outros trabalhos também se observaram elevadas freqüências de ingestão etílica entre vítimas de violência: no município de São Paulo, alcoolemia positiva foi encontrada em $42,5 \%$ das vítimas de homicídio no segundo semestre do ano de $2001^{10}$ e em 52,3\% das vítimas de homicídio no ano de 1994. ${ }^{4}$ Em Curitiba, com base em evidências clínicas (hálito etílico, agitação e incoordenação motora) e relato dos pacientes, constatou-se que 50,2\% das vítimas de agressões interpessoais, por arma de fogo ou arma branca, estavam alcoolizadas, ${ }^{12}$ e em Belo Horizonte (MG) verificou-se que, de 207 vítimas de trauma por arma branca atendidas em um pronto socorro, 59,4\% haviam ingerido bebidas alcoólicas. ${ }^{14}$

O questionário CAGE tem boa sensibilidade e especificidade para detecção de alcoolismo quando utilizado no ambiente de pronto-socorro. ${ }^{20}$ Entre os pacientes com alcoolemia positiva e entre aqueles com história positiva de ingestão etílica prévia ao trauma, 81,5\% e
82,2\%, respectivamente, tinham questionário CAGE positivo; isto mostra que a maioria dos pacientes vítimas de causas externas após ingestão etílica não era alcoolista ocasional, e sim provável usuário crônico ou dependente de álcool. A metodologia utilizada no presente estudo não rastreou o uso nocivo de bebidas alcoólicas entre os pacientes avaliados, o que constitui uma limitação deste trabalho.

Estudo multicêntrico, envolvendo sete cidades da América Latina (Salvador e Rio de Janeiro no Brasil) e Madri, na Espanha, mostrou que indivíduos mais freqüentemente vítimas da violência urbana são do sexo masculino, mais jovens e que consomem álcool. ${ }^{6} \mathrm{Um}$ exemplo de como o abuso do álcool pode aumentar a freqüência de violência foi observado no município de Diadema (SP), considerada, em 1999, uma das cidades com os maiores índices de homicídios do Brasil. Após a implantação da "lei seca”, a qual determina o fechamento de bares daquela cidade as $23 \mathrm{~h}$, registrou-se redução de $44 \%$ no índice de homicídios. ${ }^{8}$ Isto pode ser justificado pelo fato de que entre os efeitos do álcool no sistema nervoso central se incluem a perda do autocontrole e a liberação de impulsos agressivos, o que se configura em riscos para a integridade física do indivíduo e das pessoas próximas. ${ }^{19}$

A maior freqüência de necessidade de internação entre os pacientes com alcoolemia positiva, atendidos no pronto-socorro mostra que os acidentes envolvendo pessoas alcoolizadas tendem a ser mais graves. Este fato já havia sido descrito, pelo menos, em outros dois estudos, um brasileiro, realizado em São Paulo ${ }^{11}$ e outro em Los Angeles (Estados Unidos). ${ }^{7}$ O predomínio da cabeça (crânio e face) como segmento corpóreo mais afetado entre os pacientes com alcoolemia positiva ou com história de ingestão etílica prévia ao trauma pode estar relacionado, pelo menos em parte, à proporcionalmente maior freqüência de agressões físicas observada entre eles. Geralmente, vítimas de agressões físicas e/ou homicídios apresentam grandes concentrações de ferimentos na cabeça. ${ }^{10} \mathrm{~A}$ maior freqüiência de trauma raquimedular entre aqueles com história positiva de ingestão alcoólica prévia ao trauma, também mostra a potencialidade de maior gravidade dos traumas em indivíduos alcoolizados.

Entre os pacientes com alcoolemia positiva, bem como entre aqueles com história prévia de ingestão etílica, a maior freqüência de ocorrências de acidentes foi no final de semana e no período noturno, similarmente ao encontrado em outros estudos. ${ }^{5,10,11}$ Isso provavelmente se deve ao fato de que nestes períodos são mais comuns as reuniões de lazer associadas ao consumo de bebidas alcoólicas.

\footnotetext{
a Laranjeira R, Pinsky I, Zaleski M, Caetano R. I levantamento nacional sobre os padrões de consumo de álcool na população brasileira [Internet] Brasília: Secretaria Nacional Antidrogas; 2007 [citado 2008 ago 3]. Disponível em: http://www.senad.gov.br/relatorio_padroes_consumo_alcool.pdf
} 
Em conclusão, entre os pacientes vítimas de causas externas, cerca de um terço ingeriu bebidas alcoólicas antes do trauma, a maioria era etilista crônico, homens, jovens, com pouca escolaridade e com baixa renda familiar. A causa externa mais freqüente foi o acidente de trânsito. Proporcionalmente, a ingestão prévia de bebidas alcoólicas foi mais freqüente entre os pacientes vítimas de violência. As vítimas com alcoolemia positiva tiveram com maior freqüência lesões mais graves. A ocorrência de acidentes relacionada ao uso prévio de bebidas alcoólicas foi mais freqüente nos finais de semana e no período noturno.

\section{AGRADECIMENTO}

À Selma Regina Guerra Valente, biomédica do laboratório de análises clínicas do Hospital de Clínicas de Uberlândia, pela realização das determinações laboratoriais das alcoolemias. 


\section{REFERÊNCIAS}

1. Borges G, Cherpitel C, Orozco R, Bond J, Ye Y, Macdonald $S$, et al. Multicentre study of acute alcohol use and non-fatal injuries: data from the $\mathrm{WHO}$ collaborative study on alcohol and injuries. Bull World Health Organ. 2006;84(6):453-60. DOI: 10.2471/ BLT.05.027466

2. Caetano R, Laranjeira R. A 'perfect storm' in developing countries: economic growth and the alcohol industry. Addiction. 2006;101(2):149-52. DOI: 10.1111/j.1360-0443.2006.01334.x

3. Carlini EA, Galduróz JC, Noto AR, Carlini CM, Oliveira LG, Nappo AS, et al. Il levantamento domiciliar sobre o uso de drogas psicotrópicas no Brasil: estudo envolvendo as 108 maiores cidades do país: 2005. São Paulo: Páginas \& Letras; 2007.

4. Carlini-Cotrim B, Matta Chasin AA. Blood alcohol content and death from fatal injury: a study in the metropolitan area of São Paulo, Brazil. J Psychoactive Drugs. 2000;32(3):269-75.

5. Chaves AG, Pereira EA, Anicete GC, Ritt AG, Mello, AL, Nunes CA, et al. Alcoolemia em acidentados de trânsito. Rev HPS. 1989;35(1):27-30.

6. Cruz JM. La victimización por violencia urbana: niveles y factores asociados en ciudades de América Latina y España. Rev Panam Salud Publica. 1999;5(4/5):259-67.

7. Demetriades D, Gkiokas G, Velmahos GC, Brown C, Murray J, Noguchi T. Alcohol and illicit drugs in traumatic deaths: prevalence and association with type and severity of injuries. I Am Coll Surg. 2004;199(5):687-92. DOI: 10.1016/j.jamcollsurg.2004 .07 .017

8. Duailibi S, Ponicki W, Grube J, Pinsky I, Laranjeira $\mathrm{R}$, Raw M. The effect of restricting opening hours on alcohol-related violence. Am J Public Health. 2007;97(12):2276-80. DOI: 10.2105/ AJPH.2006.092684

9. Ewing JA. Detecting alcoholism. The CAGE questionnaire. JAMA. 1984;252(14):1905-7. DOI: 10.1001/jama.252.14.1905

10. Gawryszewski VP, Kahn T, Mello Jorge MHP. Informações sobre homicídios e sua integração com o setor saúde e segurança pública. Rev Saude Publica. 2005;39(4):627-33. DOI: 10.1590/S003489102005000400017
11. Gazal-Carvalho C, Carlini-Cotrim B, Silva OA, Sauaia N. Prevalência de alcoolemia em vítimas de causas externas admitidas em centro urbano de atenção ao trauma. Rev Saude Publica. 2002;36(1):47-54. DOI: 10.1590/S0034-89102002000100008

12. Karstein AA, Burin JR, Arantes RKC, Falavinha RS. Análise epidemiológica das vítimas atendidas pelo SIATE e transportadas ao Hospital Cajuru. Rev Bras Ortop. 1996;31(6):485-90.

13. Laurenti R, Mello Jorge MHP, Gotlieb SLD. Perfil epidemiológico da morbi-mortalidade masculina. Cienc Saude Colet. 2005;10(1):35-46. DOI: 10.1590/ S1413-81232005000100010

14. Leite FKS, Pisoler LT, Coelho FA, Corradi S, Alvim BR, Sander FH. O trauma por arma branca. Análise de 207 casos. Acta Cir Bras. 2003;18(Supl 3):27.

15. Marín L, Queiroz MS. A atualidade dos acidentes de trânsito na era da velocidade: uma visão geral. Cad Saude Publica. 2000;16(1):7-21.

16. Marques ACPR, Ribeiro M. Abuso e dependência do álcool. São Paulo: AMB; Brasília DF: CFM; 2002. (Projeto Diretrizes).

17. Neves DP. Alcoolismo: acusação ou diagnóstico? Cad Saude Publica. 2004;20(1):7-14.

18. Neves MM, Borges DR, Vilela MP. Concentração de etanol em bebidas alcoólicas mais consumidas no Brasil. GED. 1989;8(1):17-20.

19. Oga S. Fundamentos de toxicologia. 2 ed. São Paulo: Atheneu; 2003

20. Paz Filho GJ, Sato LJ, Tuleski MJ, Takata SY, Ranzi CCC, Saruhashi SY, et al. Emprego do questionário CAGE para detecção de transtornos de uso de álcool em pronto-socorro. Rev Assoc Med Bras. 2001;47(1):65-9. DOI: 10.1590/S0104-42302001000100032

21. Reis AD, Figlie NB, Laranjeira R. Prevalence of substance use among trauma patients treated in a Brazilian emergency room. Rev Bras Psiquiatr. 2006;28(3):191-5. DOI: 10.1590/S151644462006000300009

22. Sethi D, Racioppi F, Baumgarten I, Bertollini R. Reducing inequalities from injuries in Europe. Lancet. 2006;368(9554):2243-50. DOI: 10.1016/S01406736(06)68895-8

Financiado pelo Ministério da Educação/Coordenação de Aperfeiçoamento de Pessoal de Nível Superior (MEC/Capes) e Programa de Apoio à Pós-Graduação/ Fundação de Assistência Estudo e Pesquisa de Uberlândia (PROAP/FAEPU) (Proc. N. ${ }^{\circ}$ 23117007164/2004-98).

Artigo baseado na dissertação de mestrado de EAM de Freitas, apresentada ao Programa de Pós-Graduação em Ciências da Saúde da Faculdade de Medicina da Universidade Federal de Uberlândia, em 2007.

Resumo apresentado no VIII Congresso Brasileiro de Saúde Coletiva e XI Congresso Mundial de Saúde Coletiva, Rio de Janeiro, 2006. 